TRANSACTIONS OF THE

AMERICAN MATHEMATICAL SOCIETY

Volume 360, Number 7, July 2008, Pages 3795-3811

S 0002-9947(08)04380-8

Article electronically published on January 30, 2008

\title{
A SIMPLE FORMULA FOR AN ANALOGUE OF CONDITIONAL WIENER INTEGRALS AND ITS APPLICATIONS
}

\author{
DONG HYUN CHO
}

\begin{abstract}
Let $C[0, T]$ denote the space of real-valued continuous functions on the interval $[0, T]$ and for a partition $\tau: 0=t_{0}<t_{1}<\cdots<t_{n}=T$ of $[0, T]$, let $X_{\tau}: C[0, T] \rightarrow \mathbb{R}^{n+1}$ be given by $X_{\tau}(x)=\left(x\left(t_{0}\right), x\left(t_{1}\right), \cdots, x\left(t_{n}\right)\right)$.

In this paper, with the conditioning function $X_{\tau}$, we derive a simple formula for conditional expectations of functions defined on $C[0, T]$ which is a probability space and a generalization of Wiener space. As applications of the formula, we evaluate the conditional expectation of functions of the form

$$
F_{m}(x)=\int_{0}^{T}(x(t))^{m} d t, \quad m \in \mathbb{N},
$$

for $x \in C[0, T]$ and derive a translation theorem for the conditional expectation of integrable functions defined on the space $C[0, T]$.
\end{abstract}

\section{INTRODUCTION AND PRELIMINARIES}

Let $C_{0}[0, T]$ be the space of continuous real-valued functions $x$ on $[0, T]$ with $x(0)=0$. It is well-known that the space $C_{0}[0, T]$ equipped with Wiener measure is a probability space. On the space, Yeh introduced an inversion formula that a conditional expectation can be found by Fourier-transform (9). Also, in [10, 11, he obtained very useful results including the Kac-Feynman integral equation and the conditional Cameron-Martin translation theorem using the inversion formula. But Yeh's inversion formula is very complicated in its applications when the conditioning function is vector-valued. In [6], Park and Skoug derived a simple formula for conditional Wiener integrals on $C_{0}[0, T]$ with the conditioning function $X: C_{0}[0, T] \rightarrow \mathbb{R}^{n}$ given by

$$
X(x)=\left(x\left(t_{1}\right), \cdots, x\left(t_{n}\right)\right)
$$

where $\tau: 0=t_{0}<t_{1}<\cdots<t_{n}=T$ is a partition of the interval $[0, T]$. In their simple formula they expressed the conditional Wiener integral directly in terms of the ordinary Wiener integral. Using the formula, they generalized the Kac-Feynman formula and obtained a Cameron-Martin type translation theorem for conditional Wiener integrals.

Received by the editors May 30, 2006.

2000 Mathematics Subject Classification. Primary 28C20.

Key words and phrases. Analogue of Wiener measure, conditional Cameron-Martin translation theorem, conditional Wiener integral, simple formula for conditional $w_{\varphi}$-integral.

(C)2008 American Mathematical Society Reverts to public domain 28 years from publication 
On the other hand, let $C[0, T]$ denote the space of continuous real-valued functions on the interval $[0, T]$. Im and Ryu introduced a probability measure $w_{\varphi}$ on $(C[0, T], \mathcal{B}(C[0, T]))$ where $\mathcal{B}(C[0, T])$ denotes a Borel $\sigma$-algebra on $C[0, T]$ and $\varphi$ is a probability measure on $(\mathbb{R}, \mathcal{B}(\mathbb{R}))([4,7])$. This measure space is a generalization of Wiener space. In [4, they introduced a translation theorem of the $w_{\varphi}$-integral, which corresponds to the Cameron-Martin translation theorem on Wiener space (2]). Also, Im and Ryu evaluated the conditional $w_{\varphi}$-integral of various functions on $C[0, T]$ with the conditioning function $X_{\tau}: C[0, T] \rightarrow \mathbb{R}^{n+1}$ given by

$$
X_{\tau}(x)=\left(x\left(t_{0}\right), x\left(t_{1}\right), \cdots, x\left(t_{n}\right)\right)
$$

and derived a translation theorem of the conditional $w_{\varphi}$-integral when the conditioning function is $X_{\tau}(x)=x\left(t_{n}\right)$. But their processes were complicated in its proof.

In this paper, on the space $\left(C[0, T], \mathcal{B}(C[0, T]), w_{\varphi}\right)$, we derive a simple formula for the conditional $w_{\varphi}$-integral of functions on $C[0, T]$ with the vector-valued conditioning function $X_{\tau}$ given by (11). This formula expresses the conditional $w_{\varphi^{-}}$ integral directly in terms of the non-conditional $w_{\varphi}$-integral. As applications of the formula, we evaluate the conditional $w_{\varphi}$-integral of functions of the form

$$
F_{m}(x)=\int_{0}^{T}(x(t))^{m} d t
$$

for any positive integer $m$ and using the translation theorem of $w_{\varphi}$-integral in [4, we derive a translation theorem for the conditional $w_{\varphi}$-integral of functions on $C[0, T]$ with the conditioning function $X_{\tau}$.

Throughout this paper, let $\mathbb{C}$ and $\mathbb{C}_{+}$denote the set of complex numbers and that of complex numbers with positive real parts, respectively.

Now, we begin by introducing the probability space $\left(C[0, T], \mathcal{B}(C[0, T]), w_{\varphi}\right)$.

For a positive real $T$, let $C=C[0, T]$ be the space of all real-valued continuous functions on the closed interval $[0, T]$ with the supremum norm. For $\vec{t}=\left(t_{0}, t_{1}, \cdots, t_{n}\right)$ with $0=t_{0}<t_{1}<\cdots<t_{n} \leq T$, let $J_{\vec{t}}: C[0, T] \rightarrow \mathbb{R}^{n+1}$ be the function given by

$$
J_{\vec{t}}(x)=\left(x\left(t_{0}\right), x\left(t_{1}\right), \cdots, x\left(t_{n}\right)\right) .
$$

For $B_{j}(j=0,1, \cdots, n)$ in $\mathcal{B}(\mathbb{R})$, the subset $J_{\vec{t}}^{-1}\left(\prod_{j=0}^{n} B_{j}\right)$ of $C[0, T]$ is called an interval and let $\mathcal{I}$ be the set of all such intervals. For a probability measure $\varphi$ on $(\mathbb{R}, \mathcal{B}(\mathbb{R}))$, we let

$$
m_{\varphi}\left(J_{\vec{t}}^{-1}\left(\prod_{j=0}^{n} B_{j}\right)\right)=\int_{B_{0}} \int_{\prod_{j=1}^{n} B_{j}} W_{n+1}\left(\vec{t} ; u_{0}, u_{1}, \cdots, u_{n}\right) d\left(u_{1}, \cdots, u_{n}\right) d \varphi\left(u_{0}\right),
$$

where

$$
\begin{aligned}
& W_{n+1}\left(\vec{t} ; u_{0}, u_{1}, \cdots, u_{n}\right) \\
= & {\left[\prod_{j=1}^{n} \frac{1}{2 \pi\left(t_{j}-t_{j-1}\right)}\right]^{\frac{1}{2}} \exp \left\{-\frac{1}{2} \sum_{j=1}^{n} \frac{\left(u_{j}-u_{j-1}\right)^{2}}{t_{j}-t_{j-1}}\right\} . }
\end{aligned}
$$

$\mathcal{B}(C[0, T])$, the Borel $\sigma$-algebra of $C[0, T]$, coincides with the smallest $\sigma$-algebra generated by $\mathcal{I}$ and there exists a unique probability measure $w_{\varphi}$ on $(C[0, T], \mathcal{B}(C[0, T]))$ 
such that $w_{\varphi}(I)=m_{\varphi}(I)$ for all $I$ in $\left.\mathcal{I}(4,4,8]\right)$. This measure $w_{\varphi}$ is called an analogue of Wiener measure associated with the probability measure $\varphi$.

By the change of variable theorem, we can easily prove the following theorem.

Theorem 1.1 ([4, Lemma 2.1]). If $f: \mathbb{R}^{n+1} \rightarrow \mathbb{C}$ is a Borel measurable function, then we have

$$
\begin{aligned}
& \int_{C} f\left(x\left(t_{0}\right), x\left(t_{1}\right), \cdots, x\left(t_{n}\right)\right) d w_{\varphi}(x) \\
\stackrel{*}{=} & \int_{\mathbb{R}} \int_{\mathbb{R}^{n}} f\left(u_{0}, u_{1}, \cdots, u_{n}\right) W_{n+1}\left(\vec{t} ; u_{0}, u_{1}, \cdots, u_{n}\right) d\left(u_{1}, \cdots, u_{n}\right) d \varphi\left(u_{0}\right)
\end{aligned}
$$

where $\stackrel{*}{=}$ means that if either side exists, then both sides exist and they are equal.

Let $C_{0}=C_{0}[0, T]$ be a Wiener space with Wiener measure $m_{w}$. Then Theorem 1.1 is reduced to the well-known Wiener integration theorem if $\varphi$ is a Dirac measure concentrated at 0 .

Theorem 1.2 (Wiener integration theorem). If $f: \mathbb{R}^{n} \rightarrow \mathbb{C}$ is a Borel measurable function, then we have

$$
\begin{aligned}
& \int_{C_{0}} f\left(x\left(t_{1}\right), \cdots, x\left(t_{n}\right)\right) d m_{w}(x) \\
\stackrel{*}{=} & \int_{\mathbb{R}^{n}} f\left(u_{1}, \cdots, u_{n}\right) W_{n+1}\left(\vec{t} ; 0, u_{1}, \cdots, u_{n}\right) d\left(u_{1}, \cdots, u_{n}\right)
\end{aligned}
$$

where $\stackrel{*}{=}$ means that if either side exists, then both sides exist and they are equal.

Let $\left\{e_{k}: k=1,2, \cdots\right\}$ be a complete orthonormal subset of $L_{2}[0, T]$ such that each $e_{k}$ is of bounded variation. For $f$ in $L_{2}[0, T]$ and $x$ in $C[0, T]$, we let

$$
(f, x)=\lim _{n \rightarrow \infty} \int_{0}^{T}\left[\sum_{k=1}^{n} e_{k}(t) \int_{0}^{T} f(s) e_{k}(s) d s\right] d x(t)
$$

if the limit exists. $(f, x)$ is called the Paley-Wiener-Zygmund integral of $f$ according to $x$.

Let $F: C[0, T] \rightarrow \mathbb{C}$ be integrable and let $X$ be a random vector on $C[0, T]$. Then, we have the conditional expectation $E[F \mid X]$ of $F$ given $X$ from a wellknown probability theory ([5]). Further, there exists a $P_{X}$-integrable complexvalued function $\psi$ on the value space of $X$ such that $E[F \mid X](x)=(\psi \circ X)(x)$ for $w_{\varphi}$-a.e. $x \in C[0, T]$, where $P_{X}$ is the probability distribution of $X$. The function $\psi$ is called the conditional $w_{\varphi}$-integral of $F$ given $X$ and it is also denoted by $E[F \mid X]$.

\section{A SIMPle FORMUla FOR THE CONDitional $w_{\varphi}$-INTEGRAL}

In this section, we derive a simple formula for the conditional $w_{\varphi}$-integral of the functions on $C[0, T]$ with the conditioning function $X_{\tau}$ given by (1).

First, we define a stochastic process $X_{t}(x): C[0, T] \rightarrow \mathbb{R}$ by

$$
X_{t}(x)=x(t)
$$

for $t \in[0, T]$. We have the following lemma which is useful to prove several results. 
Lemma 2.1. Let $W_{t}$ be the standard Wiener process given by $W_{t}(x)=x(t)$ on $C_{0}[0, T]$. Then $X_{t}-X_{s}$ and $W_{t}-W_{s}$ have the same distribution if $0 \leq s \leq t \leq T$, that is, for any Borel subset $B$ of $\mathbb{R}$

$$
w_{\varphi}\left(X_{t}-X_{s} \in B\right)=m_{w}\left(W_{t}-W_{s} \in B\right)
$$

Moreover, if $0 \leq s<t \leq T$, then they are normally distributed with mean 0 and variance $t-s$. In particular, if $0 \leq s_{1}<s_{2} \leq s_{3}<s_{4} \leq T$, then $X_{s_{4}}-X_{s_{3}}$ and $X_{s_{2}}-X_{s_{1}}$ are stochastically independent on $C[0, T]$.

Proof. Suppose that $s=t$. Then for a Borel subset $B$ of $\mathbb{R}$

$$
w_{\varphi}\left(X_{t}-X_{s} \in B\right)=\delta_{0}(B)=m_{w}\left(W_{t}-W_{s} \in B\right)
$$

where $\delta_{0}$ is Dirac measure concentrated at 0 .

Now suppose that $0<s<t$. By Theorem 1.1, we have

$$
\begin{aligned}
w_{\varphi}\left(X_{t}-X_{s} \in B\right)= & \int_{C} \chi_{B}(x(t)-x(s)) d w_{\varphi}(x) \\
= & {\left[\frac{1}{(2 \pi)^{2} s(t-s)}\right]^{\frac{1}{2}} \int_{\mathbb{R}} \int_{\mathbb{R}^{2}} \chi_{B}\left(u_{2}-u_{1}\right) \exp \left\{-\frac{\left(u_{1}-u_{0}\right)^{2}}{2 s}\right.} \\
& \left.-\frac{\left(u_{2}-u_{1}\right)^{2}}{2(t-s)}\right\} d\left(u_{1}, u_{2}\right) d \varphi\left(u_{0}\right)
\end{aligned}
$$

where $\chi_{B}$ denotes the indicator function concentrated on $B$. Let $v_{2}=u_{2}-u_{1}$ and $v_{1}=u_{1}-u_{0}$. By the change of variable and Fubini's theorems, we have

$$
\begin{aligned}
w_{\varphi}\left(X_{t}-X_{s} \in B\right)= & {\left[\frac{1}{(2 \pi)^{2} s(t-s)}\right]^{\frac{1}{2}} \int_{\mathbb{R}} \int_{\mathbb{R}} \int_{\mathbb{R}} \chi_{B}\left(v_{2}\right) \exp \left\{-\frac{v_{1}^{2}}{2 s}-\frac{v_{2}^{2}}{2(t-s)}\right\} } \\
& d \varphi\left(u_{0}\right) d v_{1} d v_{2} \\
= & {\left[\frac{1}{2 \pi(t-s)}\right]^{\frac{1}{2}} \int_{B} \exp \left\{-\frac{v_{2}^{2}}{2(t-s)}\right\} d v_{2} } \\
= & m_{w}\left(W_{t}-W_{s} \in B\right)
\end{aligned}
$$

since $\varphi$ is a probability measure and $W_{t}-W_{s}$ is normally distributed with mean 0 , variance $t-s$. If $0=s<t$, the proof is similar to the case $0<s<t$.

Since $W_{s_{4}}-W_{s_{3}}$ and $W_{s_{2}}-W_{s_{1}}$ are stochastically independent on $C_{0}[0, T]$, $X_{s_{4}}-X_{s_{3}}$ and $X_{s_{2}}-X_{s_{1}}$ are stochastically independent on $C[0, T]$. Now the proof is completed.

Remark 2.2. It is well-known that the Wiener process $W_{t}$ is the standard Brownian motion process. On the other hand, $X_{t}$ need not be a Brownian motion process since $X_{0}(x)=x(0)$ can take arbitrary values. Note that $W_{t}$ is normally distributed with mean 0 and variance $t$, but $X_{t}$ need not be if $\varphi$ is not Dirac measure $\delta_{0}$. See (2.6) of [4, p. 803] and (6) of [7, p. 4930]. 
For a given partition $\tau: 0=t_{0}<t_{1}<\cdots<t_{n}=T$ of $[0, T]$ and for $x$ in $C[0, T]$, define the polygonal function $[x]$ on $[0, T]$ by

$$
[x](t)=x\left(t_{j-1}\right)+\frac{t-t_{j-1}}{t_{j}-t_{j-1}}\left(x\left(t_{j}\right)-x\left(t_{j-1}\right)\right), \quad t_{j-1} \leq t \leq t_{j}, \quad j=1, \cdots, n .
$$

Similarly, for $\vec{\xi}=\left(\xi_{0}, \xi_{1}, \cdots, \xi_{n}\right) \in \mathbb{R}^{n+1}$, define the polygonal function $[\vec{\xi}]$ on $[0, T]$ by

$$
[\vec{\xi}](t)=\xi_{j-1}+\frac{t-t_{j}}{t_{j}-t_{j-1}}\left(\xi_{j}-\xi_{j-1}\right), \quad t_{j-1} \leq t \leq t_{j-1}, \quad j=1, \cdots, n .
$$

Then both $[x]$ and $[\vec{\xi}]$ are continuous on $[0, T]$, their graphs are line segments on each subinterval $\left[t_{j-1}, t_{j}\right]$, and $[x]\left(t_{j}\right)=x\left(t_{j}\right)$ and $[\vec{\xi}]\left(t_{j}\right)=\xi_{j}$ at each $t_{j}$.

To derive a simple formula for the conditional $w_{\varphi}$-integral, we begin with letting for $t_{j-1}<t<t_{j}$

$$
\begin{aligned}
\alpha_{j}=\frac{t_{j}-t}{t_{j}-t_{j-1}}, \quad \beta_{j}=\frac{t-t_{j-1}}{t_{j}-t_{j-1}}, \quad \Gamma_{j}(t)=\frac{t_{j}-t_{j-1}}{\left(t_{j}-t\right)\left(t-t_{j-1}\right)}, \\
W_{j}(t, x)=x(t)-[x](t) \text { for } x \in C_{0}[0, T]
\end{aligned}
$$

and

$$
X_{j}(t, x)=x(t)-[x](t) \text { for } x \in C[0, T]
$$

for each $j=1, \cdots, n$.

The following lemma is useful to evaluate several conditional $w_{\varphi}$-integrals.

Lemma 2.3. Let $t \in\left(t_{j-1}, t_{j}\right)$ for some $j \in\{1, \cdots, n\}$ and $f: \mathbb{R} \rightarrow \mathbb{R}$ be a Borel measurable function. Then

$$
\int_{C_{0}} f\left(W_{j}(t, x)\right) d m_{w}(x) \stackrel{*}{=}\left[\frac{\Gamma_{j}(t)}{2 \pi}\right]^{\frac{1}{2}} \int_{\mathbb{R}} f(u) \exp \left\{-\frac{\Gamma_{j}(t)}{2} u^{2}\right\} d u
$$

where $\Gamma_{j}(t)$ is given by (2). In particular, $W_{j}(t, \cdot)$ is normally distributed with mean 0 and variance $\frac{1}{\Gamma_{j}(t)}$.

Proof. For convenience, let

$$
\Gamma=\left[\frac{1}{(2 \pi)^{2}\left(t_{j}-t\right)\left(t-t_{j-1}\right)}\right]^{\frac{1}{2}} .
$$

Note that

$$
\begin{aligned}
W_{j}(t, x) & =x(t)-x\left(t_{j-1}\right)-\frac{t-t_{j-1}}{t_{j}-t_{j-1}}\left(x\left(t_{j}\right)-x(t)+x(t)-x\left(t_{j-1}\right)\right) \\
& =\alpha_{j}\left(x(t)-x\left(t_{j-1}\right)\right)-\beta_{j}\left(x\left(t_{j}\right)-x(t)\right)
\end{aligned}
$$

where $\alpha_{j}$ and $\beta_{j}$ are given by (2). Now, for $j=2, \cdots, n$, we have

$$
\begin{aligned}
\int_{C_{0}} f\left(W_{j}(t, x)\right) d m_{w}(x) \stackrel{*}{=} & \int_{\mathbb{R}^{3}} f\left(\alpha_{j}\left(u_{2}-u_{1}\right)-\beta_{j}\left(u_{3}-u_{2}\right)\right) \\
& \times W_{4}\left(\left(0, t_{j-1}, t, t_{j}\right) ; 0, u_{1}, u_{2}, u_{3}\right) d\left(u_{1}, u_{2}, u_{3}\right)
\end{aligned}
$$

by Theorem 1.2 where $W_{4}$ is given by (2). Let

$$
v_{2}=\alpha_{j}\left(u_{2}-u_{1}\right)
$$

and

$$
v_{3}=-\beta_{j}\left(u_{3}-u_{2}\right)
$$


Then we have by the change of variable theorem

$$
\begin{aligned}
& \int_{C_{0}} f\left(W_{j}(t, x)\right) d m_{w}(x) \\
\stackrel{*}{=} & \frac{\Gamma}{\alpha_{j} \beta_{j}}\left(\frac{1}{2 \pi t_{j-1}}\right)^{\frac{1}{2}} \int_{\mathbb{R}^{3}} f\left(v_{2}+v_{3}\right) \exp \left\{-\frac{1}{2 t_{j-1}} u_{1}^{2}-\frac{1}{2 \alpha_{j}^{2}\left(t-t_{j-1}\right)} v_{2}^{2}\right. \\
& \left.-\frac{1}{2 \beta_{j}^{2}\left(t_{j}-t\right)} v_{3}^{2}\right\} d\left(u_{1}, v_{2}, v_{3}\right) \\
= & \frac{\Gamma}{\alpha_{j} \beta_{j}} \int_{\mathbb{R}^{2}} f\left(v_{2}+v_{3}\right) \exp \left\{-\frac{1}{2 \alpha_{j}^{2}\left(t-t_{j-1}\right)} v_{2}^{2}-\frac{1}{2 \beta_{j}^{2}\left(t_{j}-t\right)} v_{3}^{2}\right\} d\left(v_{2}, v_{3}\right) .
\end{aligned}
$$

Let $u=v_{2}+v_{3}$. Note that $\alpha_{j}^{2}\left(t-t_{j-1}\right)+\beta_{j}^{2}\left(t_{j}-t\right)=\frac{1}{\Gamma_{j}(t)}$ and hence

$$
1-\beta_{j}^{2} \Gamma_{j}(t)\left(t_{j}-t\right)=\alpha_{j}^{2} \Gamma_{j}(t)\left(t-t_{j-1}\right) .
$$

Again, by the change of variable theorem we have

$$
\begin{aligned}
& \int_{C_{0}} f\left(W_{j}(t, x)\right) d m_{w}(x) \\
\stackrel{*}{=} & \frac{\Gamma}{\alpha_{j} \beta_{j}} \int_{\mathbb{R}^{2}} f(u) \exp \left\{-\frac{1}{2 \alpha_{j}^{2}\left(t-t_{j-1}\right)}\left(u-v_{3}\right)^{2}-\frac{1}{2 \beta_{j}^{2}\left(t_{j}-t\right)} v_{3}^{2}\right\} d\left(u, v_{3}\right) \\
= & \frac{\Gamma}{\alpha_{j} \beta_{j}} \int_{\mathbb{R}} \int_{\mathbb{R}} f(u) \exp \left\{-\frac{1}{2 \alpha_{j}^{2}\left(t-t_{j-1}\right)} u^{2}+\frac{\beta_{j}^{2} \Gamma_{j}(t)\left(t_{j}-t\right)}{2 \alpha_{j}^{2}\left(t-t_{j-1}\right)} u^{2}\right. \\
& -\frac{1}{2 \alpha_{j}^{2} \beta_{j}^{2} \Gamma_{j}(t)\left(t_{j}-t\right)\left(t-t_{j-1}\right)}\left[v_{3}^{2}-2 \beta_{j}^{2} \Gamma_{j}(t)\left(t_{j}-t\right) u v_{3}+\beta_{j}^{4}\left(\Gamma_{j}(t)\right)^{2}\right. \\
& \left.\left.\times\left(t_{j}-t\right)^{2} u^{2}\right]\right\} d v_{3} d u \\
= & {\left[2 \pi \Gamma^{2} \Gamma_{j}(t)\left(t_{j}-t\right)\left(t-t_{j-1}\right)\right]^{\frac{1}{2}} \int_{\mathbb{R}} f(u) \exp \left\{-\frac{\alpha_{j}^{2} \Gamma_{j}(t)\left(t-t_{j-1}\right)}{2 \alpha_{j}^{2}\left(t-t_{j-1}\right)} u^{2}\right\} d u } \\
= & {\left[\frac{\Gamma_{j}(t)}{2 \pi}\right]^{\frac{1}{2}} \int_{\mathbb{R}} f(u) \exp \left\{-\frac{\Gamma_{j}(t)}{2} u^{2}\right\} d u }
\end{aligned}
$$

which is the desired result. If $j=1$, then the proof is similar to the case $j \geq 2$.

The following theorem gives an interesting observation for the process $x(t)-[x](t)$ on $C[0, T]$. In fact, $x(t)-[x](t)$ is a Brownian bridge motion on each subinterval.

Theorem 2.4. For each $j=1, \cdots, n$, let $W_{j}$ and $X_{j}$ be given by (3) and (44), respectively. Then $X_{j}$ is a Brownian bridge motion process on $\left[t_{j-1}, t_{j}\right]$. Moreover for $t \in\left(t_{j-1}, t_{j}\right)$, both $W_{j}(t, \cdot)$ and $X_{j}(t, \cdot)$ are normally distributed with mean 0 and variance $\frac{1}{\Gamma_{j}(t)}$ where $\Gamma_{j}(t)$ is given by (2).

Proof. Note that $W_{j}$ is a Brownian bridge motion process ([6, Theorem 1]). By Lemma 2.1. $W_{j}$ and $X_{j}$ have the same distribution so that $X_{j}$ is a Brownian bridge motion process on the subinterval $\left[t_{j-1}, t_{j}\right]$ since $X_{j}\left(t_{j-1}, x\right)=x\left(t_{j-1}\right)-[x]\left(t_{j-1}\right)=$ $0=x\left(t_{j}\right)-[x]\left(t_{j}\right)=X_{j}\left(t_{j}, x\right)$ for $x \in C[0, T]$.

The second result immediately follows from Lemmas 2.1 and 2.3 . 
By Theorem 2.4 and [6, Corollary 1], we have the following corollary.

Corollary 2.5. The processes $\left\{X_{j}(t, \cdot): t_{j-1} \leq t \leq t_{j}\right\}$, where $j=1, \cdots, n$ are stochastically independent.

Theorem 2.6. Let $Y_{\tau}: C[0, T] \rightarrow \mathbb{R}^{n+1}$ be given by

$$
Y_{\tau}(x)=\left(x\left(t_{0}\right), x\left(t_{1}\right)-x\left(t_{0}\right), \cdots, x\left(t_{n}\right)-x\left(t_{0}\right)\right) .
$$

Then the process $\{x(t)-[x](t): 0 \leq t \leq T\}$ and $Y_{\tau}$ are independent.

Proof. Define $Y:[0, T] \times C[0, T] \rightarrow \mathbb{R}$ by

$$
Y(s, x)=X_{s}(x)-X_{0}(x)=x(s)-x\left(t_{0}\right)
$$

where $X_{s}$ and $X_{0}$ are given by (11) with replacing $t$ by $s$ and 0 , respectively.

Fix $j \in\{1, \cdots, n\}$ and let $X_{j}$ be given by (44). By Theorem 2.4, for $t_{j-1}<t<t_{j}$, $X_{j}(t, \cdot)$ is normally distributed with mean 0 and so is $Y(s, \cdot)$ for $s \in\left(0, t_{j-1}\right] \cup\left[t_{j}, T\right]$ by Lemma 2.1. Moreover, it is not difficult to show that the distributions of $Y(0, \cdot)$, $X_{j}\left(t_{j-1}, \cdot\right)$ and $X_{j}\left(t_{j}, \cdot\right)$ are the Dirac measure $\delta_{0}$ concentrated at 0 .

First, we show that $X_{j}(t, \cdot)$ and $Y(s, \cdot)$ are stochastically independent for $t \in$ $\left[t_{j-1}, t_{j}\right]$ and $s \in\left[0, t_{j-1}\right] \cup\left[t_{j}, T\right]$, and so are $X_{j}(t, \cdot)$ and $X_{0}$. To prove the assertions, let $B_{1}$ and $B_{2}$ be Borel subsets of $\mathbb{R}$.

Suppose that $t=t_{j-1}$ or $t=t_{j}$. Then we have for $s \in\left[0, t_{j-1}\right] \cup\left[t_{j}, T\right]$

$$
\begin{aligned}
w_{\varphi}\left(X_{j}(t, \cdot) \in B_{1}, Y(s, \cdot) \in B_{2}\right) & =\delta_{0}\left(B_{1}\right) w_{\varphi}\left(Y(s, \cdot) \in B_{2}\right) \\
& =w_{\varphi}\left(X_{j}(t, \cdot) \in B_{1}\right) w_{\varphi}\left(Y(s, \cdot) \in B_{2}\right)
\end{aligned}
$$

which shows the independence of $X_{j}(t, \cdot)$ and $Y(s, \cdot)$. If we replace $Y(s, \cdot)$ by $X_{0}$, then we have the independence of $X_{j}(t, \cdot)$ and $X_{0}$.

Similarly, we can prove the independence of $X_{j}(t, \cdot)$ and $Y(0, \cdot)$ for $t_{j-1}<t<t_{j}$.

Now suppose that $t_{j-1}<t<t_{j}$. If $s \in\left(0, t_{j-1}\right]$, then we have

$$
\begin{aligned}
\operatorname{Cov}\left(X_{j}(t, \cdot), Y(s, \cdot)\right) & =E\left[X_{j}(t, \cdot) Y(s, \cdot)\right] \\
& =E\left[\left(\alpha_{j}\left(X_{t}-X_{t_{j-1}}\right)-\beta_{j}\left(X_{t_{j}}-X_{t}\right)\right)\left(X_{s}-X_{0}\right)\right]=0
\end{aligned}
$$

by Lemma 2.1 where $\alpha_{j}$ and $\beta_{j}$ are given by (2). If $s \in\left[t_{j}, T\right]$, then we also have

$$
\begin{aligned}
\operatorname{Cov}\left(X_{j}(t, \cdot), Y(s, \cdot)\right) & =E\left[X_{j}(t, \cdot) Y(s, \cdot)\right] \\
& =E\left[\alpha_{j}\left(X_{t}-X_{t_{j-1}}\right)^{2}-\beta_{j}\left(X_{t_{j}}-X_{t}\right)^{2}\right] \\
& =\alpha_{j}\left(t-t_{j-1}\right)-\beta_{j}\left(t_{j}-t\right)=0
\end{aligned}
$$

by Lemma 2.1, which shows the independence of $X_{j}(t, \cdot)$ and $Y(s, \cdot)$. Finally, let

$$
\Delta_{t}=\left[\frac{1}{(2 \pi)^{3}\left(t_{j}-t\right)\left(t-t_{j-1}\right) t_{j-1}}\right]^{\frac{1}{2}},
$$

if $j \in\{2, \cdots, n\}$. Then we have by Theorem 1.1

$$
\begin{aligned}
& w_{\varphi}\left(X_{j}(t, \cdot) \in B_{1}, X_{0} \in B_{2}\right) \\
= & \int_{C} \chi_{B_{1}}\left(X_{j}(t, x)\right) \chi_{B_{2}}\left(X_{0}(x)\right) d w_{\varphi}(x) \\
= & \Delta_{t} \int_{\mathbb{R}} \int_{\mathbb{R}^{3}} \chi_{B_{1}}\left(\alpha_{j}\left(u_{2}-u_{1}\right)-\beta_{j}\left(u_{3}-u_{2}\right)\right) \chi_{B_{2}}\left(u_{0}\right) \exp \left\{-\frac{\left(u_{1}-u_{0}\right)^{2}}{2 t_{j-1}}\right. \\
& \left.-\frac{\left(u_{2}-u_{1}\right)^{2}}{2\left(t-t_{j-1}\right)}-\frac{\left(u_{3}-u_{2}\right)^{2}}{2\left(t_{j}-t\right)}\right\} d\left(u_{1}, u_{2}, u_{3}\right) d \varphi\left(u_{0}\right) .
\end{aligned}
$$


For $i=1,2,3$, let $v_{i}=u_{i}-u_{i-1}$. By the change of variable theorem and Fubini's theorem, we have

$$
\begin{aligned}
& w_{\varphi}\left(X_{j}(t, \cdot) \in B_{1}, X_{0} \in B_{2}\right) \\
= & \Delta_{t} \int_{\mathbb{R}} \int_{\mathbb{R}^{3}} \chi_{B_{1}}\left(\alpha_{j} v_{2}-\beta_{j} v_{3}\right) \chi_{B_{2}}\left(u_{0}\right) \exp \left\{-\frac{v_{1}^{2}}{2 t_{j-1}}-\frac{v_{2}^{2}}{2\left(t-t_{j-1}\right)}-\frac{v_{3}^{2}}{2\left(t_{j}-t\right)}\right\} \\
& d\left(v_{1}, v_{2}, v_{3}\right) d \varphi\left(u_{0}\right) \\
= & {\left[\Delta_{t} \int_{\mathbb{R}} \int_{\mathbb{R}^{3}} \chi_{B_{1}}\left(\alpha_{j} v_{2}-\beta_{j} v_{3}\right) \exp \left\{-\frac{v_{1}^{2}}{2 t_{j-1}}-\frac{v_{2}^{2}}{2\left(t-t_{j-1}\right)}-\frac{v_{3}^{2}}{2\left(t_{j}-t\right)}\right\}\right.} \\
& \left.d\left(v_{1}, v_{2}, v_{3}\right) d \varphi\left(u_{0}\right)\right]\left[\int_{\mathbb{R}} \chi_{B_{2}}\left(u_{0}\right) d \varphi\left(u_{0}\right)\right] \\
= & {\left[\Delta _ { t } \int _ { \mathbb { R } } \int _ { \mathbb { R } ^ { 3 } } \chi _ { B _ { 1 } } ( \alpha _ { j } ( u _ { 2 } - u _ { 1 } ) - \beta _ { j } ( u _ { 3 } - u _ { 2 } ) ) \operatorname { e x p } \left\{-\frac{\left(u_{1}-u_{0}\right)^{2}}{2 t_{j-1}}-\frac{\left(u_{2}-u_{1}\right)^{2}}{2\left(t-t_{j-1}\right)}\right.\right.} \\
& \left.\left.-\frac{\left(u_{3}-u_{2}\right)^{2}}{2\left(t_{j}-t\right)}\right\} d\left(u_{1}, u_{2}, u_{3}\right) d \varphi\left(u_{0}\right)\right]\left[\int_{\mathbb{R}} \chi_{B_{2}}\left(u_{0}\right) d \varphi\left(u_{0}\right)\right] \\
= & w_{\varphi}\left(X_{j}(t, \cdot) \in B_{1}\right) w_{\varphi}\left(X_{0} \in B_{2}\right)
\end{aligned}
$$

which shows the independence of $X_{j}(t, \cdot)$ and $X_{0}$. If $j=1$, then we can show that $X_{j}(t, \cdot)$ and $X_{0}$ are independent by a similar method.

Now, for each $j=1, \cdots, n$, the process $\left\{X_{j}(t, \cdot): t_{j-1} \leq t \leq t_{j}\right\}$ is independent of $Y_{\tau}$, which completes the proof.

Remark 2.7. Since for $x \in C[0, T]$ and $t_{j-1} \leq t \leq t_{j}$

$$
\begin{aligned}
X_{j}(t, x)= & \alpha_{j}\left(x(t)-x\left(t_{j-1}\right)\right)-\beta_{j}\left(x\left(t_{j}\right)-x(t)\right) \\
= & \alpha_{j}\left(x(t)-x\left(t_{0}\right)-\left(x\left(t_{j-1}\right)-x\left(t_{0}\right)\right)\right) \\
& -\beta_{j}\left(x\left(t_{j}\right)-x\left(t_{0}\right)-\left(x(t)-x\left(t_{0}\right)\right)\right),
\end{aligned}
$$

we can also obtain Corollary 2.5 from Theorem 2.6 .

Theorem 2.8. Let $X_{\tau}: C[0, T] \rightarrow \mathbb{R}^{n+1}$ be given by

$$
X_{\tau}(x)=\left(x\left(t_{0}\right), x\left(t_{1}\right), \cdots, x\left(t_{n}\right)\right) .
$$

Then the process $\{x(t)-[x](t): 0 \leq t \leq T\}$ and $X_{\tau}$ are independent.

Proof. Take $j \in\{1, \cdots, n\}$. For $s \in\left[0, t_{j-1}\right] \cup\left[t_{j}, T\right]$, we have

$$
X_{s}(x)=x(s)=\left(x(s)-x\left(t_{0}\right)\right)+x\left(t_{0}\right) .
$$

Note that $X_{j}(t, x)=x(t)-[x](t), t_{j-1} \leq t \leq t_{j}$ and $\left(x(s)-x\left(t_{0}\right), x\left(t_{0}\right)\right)$ are independent by Theorem 2.6. Define $g: \mathbb{R}^{2} \rightarrow \mathbb{R}$ by $g\left(u_{1}, u_{2}\right)=u_{1}+u_{2}$. Since $g$ is Borel measurable and $x(s)=g\left(x(s)-x\left(t_{0}\right), x\left(t_{0}\right)\right), X_{j}(t, \cdot)$ and $X_{s}$ are independent. Now the theorem follows.

Applying the same method used in the proof of Theorem 2 in [6, p. 383] with Problem 4 of [1, p. 216], we have the following theorem from Theorem 2.8 .

Theorem 2.9. Let $F: C[0, T] \rightarrow \mathbb{C}$ be integrable and $X_{\tau}$ be given by (5) of Theorem 2.8. Then for a Borel subset $B$ of $\mathbb{R}^{n+1}$ we have

$$
\int_{X_{\tau}^{-1}(B)} F(x) d w_{\varphi}(x)=\int_{B} E[F(x-[x]+[\vec{\xi}])] d P_{X_{\tau}}(\vec{\xi})
$$

where $P_{X_{\tau}}$ is the probability distribution of $X_{\tau}$ on $\left(\mathbb{R}^{n+1}, \mathcal{B}\left(\mathbb{R}^{n+1}\right)\right)$. 
Remark 2.10. We emphasize that both $[x]\left(t_{0}\right)=x\left(t_{0}\right)$ and $[\vec{\xi}]\left(t_{0}\right)=\xi_{0}$ need not be 0 . Applying the same method used in the proof of Theorem 2 in [6, p. 383], we can prove (6) using Theorem 2.6 directly.

For a function $F: C[0, T] \rightarrow \mathbb{C}$ and $\lambda>0$, let $F^{\lambda}(x)=F\left(\lambda^{-\frac{1}{2}} x\right)$ and $X_{\tau}^{\lambda}(x)=$ $X_{\tau}\left(\lambda^{-\frac{1}{2}} x\right)$. Suppose that $E\left[F^{\lambda}\right]$ exists for each $\lambda>0$. By the definition of the conditional $w_{\varphi}$-integral and ([6), we have

$$
E\left[F^{\lambda} \mid X_{\tau}^{\lambda}\right](\vec{\xi})=E\left[F\left(\lambda^{-\frac{1}{2}}(x-[x])+[\vec{\xi}]\right)\right]
$$

for $P_{X_{\tau}^{\lambda}}$-a.e. $\vec{\xi} \in \mathbb{R}^{n+1}$, where $P_{X_{\tau}^{\lambda}}$ is the probability distribution of $X_{\tau}^{\lambda}$ on $\left(\mathbb{R}^{n+1}, \mathcal{B}\left(\mathbb{R}^{n+1}\right)\right)$. If $E\left[F\left(\lambda^{-\frac{1}{2}}(x-[x])+[\vec{\xi}]\right)\right]$ has the analytic extension $J_{\lambda}^{*}(F)(\vec{\xi})$ on $\mathbb{C}_{+}$as a function of $\lambda$, then it is denoted by

$$
E^{a n w_{\lambda}}\left[F \mid X_{\tau}\right](\vec{\xi})=J_{\lambda}^{*}(F)(\vec{\xi})
$$

for $\vec{\xi} \in \mathbb{R}^{n+1}$. Moreover, if for a non-zero real $q, E^{a n w_{\lambda}}\left[F \mid X_{\tau}\right](\vec{\xi})$ has a limit as $\lambda$ approaches to $-i q$ through $\mathbb{C}_{+}$, then it is denoted by

$$
E^{a n f_{q}}\left[F \mid X_{\tau}\right](\vec{\xi})=\lim _{\lambda \rightarrow-i q} E^{a n w_{\lambda}}\left[F \mid X_{\tau}\right](\vec{\xi}) .
$$

\section{Evaluations of CONDitional $w_{\varphi}$-INTEGRALS}

Throughout this section, let $X_{\tau}$ be given by (5) and $P_{X_{\tau}}$ denote the probability distribution of $X_{\tau}$ on $\left(\mathbb{R}^{n+1}, \mathcal{B}\left(\mathbb{R}^{n+1}\right)\right)$. Now, we evaluate conditional $w_{\varphi}$-integrals of functions on $C[0, T]$ as applications of ([6) in Theorem [2.9.

Theorem 3.1. Let $F_{m}(x)=\int_{0}^{T}(x(t))^{m} d t(m \in \mathbb{N})$ for $x \in C[0, T]$ and suppose that $\int_{\mathbb{R}}|u|^{m} d \varphi(u)<\infty$. Then $F_{m}$ is $w_{\varphi}$-integrable. Moreover, $E\left[F_{m} \mid X_{\tau}\right](\vec{\xi})$ exists for $P_{X_{\tau}}$-a.e. $\vec{\xi} \in \mathbb{R}^{n+1}$ and it is given by

$$
E\left[F_{m} \mid X_{\tau}\right](\vec{\xi})=\sum_{j=1}^{n} \sum_{k=0}^{M} \frac{m !}{2^{k} k !(m-2 k) !} \int_{t_{j-1}}^{t_{j}}([\vec{\xi}](t))^{m-2 k}\left[\frac{1}{\Gamma_{j}(t)}\right]^{k} d t
$$

where $\Gamma_{j}(t)$ is given by (2) and $M=\frac{m}{2}$ if $m$ is even and $M=\frac{m-1}{2}$ if $m$ is odd.

Proof. First, we show that $F_{m}$ is $w_{\varphi}$-integrable. Indeed, we have

$$
\begin{aligned}
\int_{C}\left|F_{m}(x)\right| d w_{\varphi}(x) & \leq \int_{0}^{T} \int_{C}|x(t)|^{m} d w_{\varphi}(x) d t \\
& =\int_{(0, T]}\left(\frac{1}{2 \pi t}\right)^{\frac{1}{2}} \int_{\mathbb{R}} \int_{\mathbb{R}}\left|u_{1}\right|^{m} \exp \left\{-\frac{\left(u_{1}-u_{0}\right)^{2}}{2 t}\right\} d u_{1} d \varphi\left(u_{0}\right) d t \\
& =\int_{(0, T]}\left(\frac{1}{2 \pi t}\right)^{\frac{1}{2}} \int_{\mathbb{R}} \int_{\mathbb{R}}\left|v+u_{0}\right|^{m} \exp \left\{-\frac{v^{2}}{2 t}\right\} d v d \varphi\left(u_{0}\right) d t
\end{aligned}
$$


by the change of variable theorem and Theorem 1.1. where $v=u_{1}-u_{0}$. By the binomial expansion, we have

$$
\begin{aligned}
& \int_{C}\left|F_{m}(x)\right| d w_{\varphi}(x) \\
\leq & \int_{(0, T]}\left[\sum_{l=0}^{m}\left(\begin{array}{c}
m \\
l
\end{array}\right)\left(\frac{1}{2 \pi t}\right)^{\frac{1}{2}} \int_{\mathbb{R}} \int_{\mathbb{R}}|v|^{l}\left|u_{0}\right|^{m-l} \exp \left\{-\frac{v^{2}}{2 t}\right\} d v d \varphi\left(u_{0}\right)\right] d t \\
= & 2 \int_{(0, T]}\left[\sum_{l=0}^{m}\left(\begin{array}{c}
m \\
l
\end{array}\right)\left[\left(\frac{1}{2 \pi t}\right)^{\frac{1}{2}} \int_{0}^{\infty} v^{l} \exp \left\{-\frac{v^{2}}{2 t}\right\} d v\right]\left[\int_{\mathbb{R}}\left|u_{0}\right|^{m-l} d \varphi\left(u_{0}\right)\right]\right] d t .
\end{aligned}
$$

Now, for $l=0,1, \cdots, m$, we have

$$
\begin{aligned}
\int_{\mathbb{R}}\left|u_{0}\right|^{m-l} d \varphi\left(u_{0}\right) & =\int_{\left|u_{0}\right| \leq 1}\left|u_{0}\right|^{m-l} d \varphi\left(u_{0}\right)+\int_{\left|u_{0}\right|>1}\left|u_{0}\right|^{m-l} d \varphi\left(u_{0}\right) \\
& \leq \int_{\left|u_{0}\right| \leq 1} d \varphi\left(u_{0}\right)+\int_{\left|u_{0}\right|>1}\left|u_{0}\right|^{m} d \varphi\left(u_{0}\right) \\
& \leq \int_{\left|u_{0}\right| \leq 1} d \varphi\left(u_{0}\right)+\int_{\mathbb{R}}\left|u_{0}\right|^{m} d \varphi\left(u_{0}\right) \equiv K<\infty
\end{aligned}
$$

by assumption. Let $u=\frac{v^{2}}{2 t}$. Then $v=(2 t u)^{\frac{1}{2}}$ and hence by the change of variable theorem it follows that

$$
\begin{aligned}
\int_{C}\left|F_{m}(x)\right| d w_{\varphi}(x) & \leq K\left(\frac{1}{\pi}\right)^{\frac{1}{2}} \int_{(0, T]}\left[\sum_{l=0}^{m}\left(\begin{array}{c}
m \\
l
\end{array}\right)(2 t)^{\frac{l}{2}} \int_{0}^{\infty} u^{\frac{l+1}{2}-1} \exp \{-u\} d u\right] d t \\
& =K\left(\frac{1}{\pi}\right)^{\frac{1}{2}} \sum_{l=0}^{m}\left(\begin{array}{c}
m \\
l
\end{array}\right) \frac{(2 T)^{\frac{l}{2}+1}}{l+2} \Gamma\left(\frac{l+1}{2}\right)<\infty
\end{aligned}
$$

where $\Gamma$ denotes the gamma function. Now, by Theorem 2.9, we have for $P_{X_{\tau}}$-a.e. $\vec{\xi} \in \mathbb{R}^{n+1}$

$$
\begin{aligned}
E\left[F_{m} \mid X_{\tau}\right](\vec{\xi}) & \\
& =\int_{0}^{T} \int_{C}(x(t)-[x](t)+[\vec{\xi}](t))^{m} d w_{\varphi}(x) d t \\
& =\sum_{j=1}^{n} \int_{t_{j-1}}^{t_{j}} \int_{C}\left(X_{j}(t, x)+[\vec{\xi}](t)\right)^{m} d w_{\varphi}(x) d t \\
& =\sum_{j=1}^{n} \int_{t_{j-1}}^{t_{j}} \int_{C}\left[\sum_{l=0}^{m}\left(\begin{array}{c}
m \\
l
\end{array}\right)\left(X_{j}(t, x)\right)^{l}([\vec{\xi}](t))^{m-l}\right] d w_{\varphi}(x) d t \\
& =\sum_{j=1}^{n} \int_{t_{j-1}}^{t_{j}}\left[\sum_{l=0}^{m}\left(\begin{array}{c}
m \\
l
\end{array}\right)([\vec{\xi}](t))^{m-l}\left[\frac{\Gamma_{j}(t)}{2 \pi}\right]^{\frac{1}{2}} \int_{\mathbb{R}} u^{l} \exp \left\{-\frac{\Gamma_{j}(t)}{2} u^{2}\right\} d u\right] d t
\end{aligned}
$$


by Lemma 2.3 and Theorem 2.4 where $\Gamma_{j}(t)$ and $X_{j}$ are given by (2) and (4), respectively. Let $M=\frac{m}{2}$ if $m$ is even and $M=\frac{m-1}{2}$ if $m$ is odd. Then we have

$$
\begin{aligned}
E\left[F_{m} \mid X_{\tau}\right](\vec{\xi})= & 2 \sum_{j=1}^{n} \int_{t_{j-1}}^{t_{j}}\left[\sum_{k=0}^{M}\left(\begin{array}{c}
m \\
2 k
\end{array}\right)([\vec{\xi}](t))^{m-2 k}\left[\frac{\Gamma_{j}(t)}{2 \pi}\right]^{\frac{1}{2}} \int_{0}^{\infty} u^{2 k}\right. \\
& \left.\times \exp \left\{-\frac{\Gamma_{j}(t)}{2} u^{2}\right\} d u\right] d t \\
= & \left(\frac{1}{\pi}\right)^{\frac{1}{2}} \sum_{j=1}^{n} \sum_{k=0}^{M}\left(\begin{array}{c}
m \\
2 k
\end{array}\right) \Gamma\left(\frac{2 k+1}{2}\right) \int_{t_{j-1}}^{t_{j}}([\vec{\xi}](t))^{m-2 k}\left[\frac{2}{\Gamma_{j}(t)}\right]^{k} d t \\
= & \sum_{j=1}^{n} \sum_{k=0}^{M} \frac{m !}{2^{k} k !(m-2 k) !} \int_{t_{j-1}}^{t_{j}}([\vec{\xi}](t))^{m-2 k}\left[\frac{1}{\Gamma_{j}(t)}\right]^{k} d t
\end{aligned}
$$

which is the desired result.

Remark 3.2. The integrand in the result of Theorem 3.1 is a polynomial of degree $m$ with respect to $t$ so that the integral can always be evaluated.

In the following example, we evaluate $E\left[F_{m} \mid X_{\tau}\right], m=1,2,3$, as applications of Theorem 3.1 .

Example 3.3. Let $F_{1}(x)=\int_{0}^{T} x(t) d t$ for $x \in C[0, T]$ and suppose that $\int_{\mathbb{R}}|u| d \varphi(u)$ $<\infty$. Then for $P_{X_{\tau}}$-a.e. $\vec{\xi}=\left(\xi_{0}, \xi_{1}, \cdots, \xi_{n}\right) \in \mathbb{R}^{n+1}$, we have

$$
\begin{aligned}
E\left[F_{1} \mid X_{\tau}\right](\vec{\xi}) & =\sum_{j=1}^{n} \int_{t_{j-1}}^{t_{j}}\left[\frac{\xi_{j}-\xi_{j-1}}{t_{j}-t_{j-1}}\left(t-t_{j-1}\right)+\xi_{j-1}\right] d t \\
& =\frac{1}{2} \sum_{j=1}^{n}\left(t_{j}-t_{j-1}\right)\left(\xi_{j}+\xi_{j-1}\right)
\end{aligned}
$$

which can also be obtained by an application of Corollary 4.5 in [4].

Let $F_{2}(x)=\int_{0}^{T}(x(t))^{2} d t$ for $x \in C[0, T]$ and suppose that $\int_{\mathbb{R}} u^{2} d \varphi(u)<\infty$. Then for $P_{X_{\tau}}$-a.e. $\vec{\xi}=\left(\xi_{0}, \xi_{1}, \cdots, \xi_{n}\right) \in \mathbb{R}^{n+1}$, we have

$$
\begin{aligned}
E\left[F_{2} \mid X_{\tau}\right](\vec{\xi}) & =\sum_{j=1}^{n} \int_{t_{j-1}}^{t_{j}}\left[\frac{\left(t_{j}-t\right)\left(t-t_{j-1}\right)}{t_{j}-t_{j-1}}+\left[\frac{\xi_{j}-\xi_{j-1}}{t_{j}-t_{j-1}}\left(t-t_{j-1}\right)+\xi_{j-1}\right]^{2}\right] d t \\
& =\frac{1}{6} \sum_{j=1}^{n}\left(t_{j}-t_{j-1}\right)\left(t_{j}-t_{j-1}+2 \xi_{j}^{2}+2 \xi_{j} \xi_{j-1}+2 \xi_{j-1}^{2}\right)
\end{aligned}
$$

which is the result given by Corollary 4.10 of 4 . 
Let $F_{3}(x)=\int_{0}^{T}(x(t))^{3} d t$ for $x \in C[0, T]$ and suppose that $\int_{\mathbb{R}}|u|^{3} d \varphi(u)<\infty$. Then for $P_{X_{\tau}}$-a.e. $\vec{\xi}=\left(\xi_{0}, \xi_{1}, \cdots, \xi_{n}\right) \in \mathbb{R}^{n+1}$, we have

$$
\begin{aligned}
E\left[F_{3} \mid X_{\tau}\right](\vec{\xi})= & \sum_{j=1}^{n} \int_{t_{j-1}}^{t_{j}}\left[\frac{3\left(t_{j}-t\right)\left(t-t_{j-1}\right)}{t_{j}-t_{j-1}}\left[\frac{\xi_{j}-\xi_{j-1}}{t_{j}-t_{j-1}}\left(t-t_{j-1}\right)+\xi_{j-1}\right]\right. \\
& \left.+\left[\frac{\xi_{j}-\xi_{j-1}}{t_{j}-t_{j-1}}\left(t-t_{j-1}\right)+\xi_{j-1}\right]^{3}\right] d t \\
= & \frac{1}{2} \sum_{j=1}^{n} \xi_{j-1}\left(t_{j}-t_{j-1}\right)^{2}+\frac{1}{4} \sum_{j=1}^{n}\left(t_{j}-t_{j-1}\right)\left(\xi_{j}^{3}+\xi_{j}^{2} \xi_{j-1}+\xi_{j} \xi_{j-1}^{2}\right. \\
& \left.+\xi_{j-1}^{3}\right)+3 \sum_{j=1}^{n} \frac{\xi_{j}-\xi_{j-1}}{\left(t_{j}-t_{j-1}\right)^{2}} \int_{t_{j-1}}^{t_{j}}\left(t_{j}-t\right)\left(t-t_{j-1}\right)^{2} d t \\
= & \frac{1}{4} \sum_{j=1}^{n}\left(t_{j}-t_{j-1}\right)\left[\left(t_{j}-t_{j-1}\right)\left(\xi_{j}+\xi_{j-1}\right)+\xi_{j}^{3}+\xi_{j}^{2} \xi_{j-1}+\xi_{j} \xi_{j-1}^{2}\right. \\
& \left.+\xi_{j-1}^{3}\right]
\end{aligned}
$$

from the integration by parts formula.

Corollary 3.4. Under the assumptions and notations given as in Theorem 3.1, $E^{\text {anw }}{ }_{\lambda}\left[F_{m} \mid X_{\tau}\right](\vec{\xi})$ exists for $\lambda \in \mathbb{C}_{+}$and for $\vec{\xi} \in \mathbb{R}^{n+1}$. Moreover, for a non-zero real $q, E^{a n f_{q}}\left[F_{m} \mid X_{\tau}\right](\vec{\xi})$ exists for $\vec{\xi} \in \mathbb{R}^{n+1}$ and it is given by

$$
E^{a n f_{q}}\left[F_{m} \mid X_{\tau}\right](\vec{\xi})=\sum_{j=1}^{n} \sum_{k=0}^{M}\left(\frac{i}{q}\right)^{k} \frac{m !}{2^{k} k !(m-2 k) !} \int_{t_{j-1}}^{t_{j}}([\vec{\xi}](t))^{m-2 k}\left[\frac{1}{\Gamma_{j}(t)}\right]^{k} d t .
$$

Proof. For $\lambda>0$, we have

$E\left[F\left(\lambda^{-\frac{1}{2}}(x-[x])+[\vec{\xi}]\right)\right]=\sum_{j=1}^{n} \sum_{k=0}^{M} \frac{1}{\lambda^{k}} \frac{m !}{2^{k} k !(m-2 k) !} \int_{t_{j-1}}^{t_{j}}([\vec{\xi}](t))^{m-2 k}\left[\frac{1}{\Gamma_{j}(t)}\right]^{k} d t$

by an application of Theorem 3.1, where $M=\frac{m}{2}$ if $m$ is even and $M=\frac{m-1}{2}$ if $m$ is odd. Now, we have the corollary immediately.

Now, we generalize the result in Example 4 of [6], which is also considered by Chang and Chang in [3].

Theorem 3.5. Let $F(x)=\exp \left\{\int_{0}^{T} x(t) d t\right\}$ for $x \in C[0, T]$ and suppose that $F$ is $w_{\varphi}$-integrable. Then, for a.e. $y \in C[0, T]$, we have

$$
\lim _{\|\tau\| \rightarrow 0} E\left[F \mid X_{\tau}\right]\left(X_{\tau}(y)\right)=F(y) .
$$

Proof. For a.e. $y \in C[0, T]$, we have

$$
\begin{aligned}
E\left[F \mid X_{\tau}\right]\left(X_{\tau}(y)\right)= & \int_{C} F(x-[x]+[y]) d w_{\varphi}(x) \\
= & \exp \left\{\frac{1}{2} \sum_{j=1}^{n}\left(t_{j}-t_{j-1}\right)\left(y\left(t_{j-1}\right)+y\left(t_{j}\right)\right)\right\} \\
& \times \int_{C} \exp \left\{\int_{0}^{T}(x(t)-[x](t)) d t\right\} d w_{\varphi}(x)
\end{aligned}
$$


by Theorem 2.9 and the same process used in Example 3.3. Letting $\|\tau\| \rightarrow 0$, we have

$$
\begin{aligned}
\lim _{\|\tau\| \rightarrow 0} E\left[F \mid X_{\tau}\right]\left(X_{\tau}(y)\right)= & \lim _{\|\tau\| \rightarrow 0} \exp \left\{\frac{1}{2} \sum_{j=1}^{n}\left(t_{j}-t_{j-1}\right)\left(y\left(t_{j-1}\right)+y\left(t_{j}\right)\right)\right\} \\
& \times \int_{C} \exp \left\{\int_{0}^{T}(x(t)-[x](t)) d t\right\} d w_{\varphi}(x)=F(y)
\end{aligned}
$$

because $\lim _{\|\tau\| \rightarrow 0}(x(t)-[x](t))=0$ for $x \in C[0, T]$.

\section{A translation theorem for the Conditional $w_{\varphi}$-Integral}

In this section, we derive a translation theorem for the conditional $w_{\varphi}$-integral, which is a generalization of [6, Theorem 4]. To derive the theorem, we need a translation theorem for the $w_{\varphi}$-integral on $C[0, T]$.

Theorem 4.1 (4 , Theorem 3.1]). Let $h$ be in $C[0, T]$ and of bounded variation. Let $\alpha \in \mathbb{R}$ and let $x_{0}(t)=\int_{0}^{t} h(s) d s+\alpha$ for $0 \leq t \leq T$ and let $\varphi_{\alpha}$ be a measure on $(\mathbb{R}, \mathcal{B}(\mathbb{R}))$ such that $\varphi_{\alpha}(B)=\varphi(B+\alpha)$ for $B \in \mathcal{B}(\mathbb{R})$. Moreover, let $F$ be a measurable function on $C[0, T]$. Then, $F\left(x+x_{0}\right)$ is also measurable on $C[0, T]$ and we have

$$
\int_{C} F(x) d w_{\varphi}(x) \stackrel{*}{=} \exp \left\{-\frac{1}{2}\|h\|_{2}^{2}\right\} \int_{C} F\left(x+x_{0}\right) \exp \{-(h, x)\} d w_{\varphi_{\alpha}}(x),
$$

where $\stackrel{*}{=}$ means that if one side exists, then both sides exist and they are equal.

Let $E_{w_{\varphi}}\left[F \mid X_{\tau}\right]$ and $E_{w_{\varphi_{\alpha}}}\left[F \mid X_{\tau}\right]$ denote the conditional $w_{\varphi^{-}}$-integral and the conditional $w_{\varphi_{\alpha}}$-integral of $F$ given $X_{\tau}$, respectively. In the following theorem we derive a translation theorem for the conditional $w_{\varphi}$-integral using Theorem 4.1 ,

Theorem 4.2. Let $x_{0}$ and $\varphi_{\alpha}$ be given as in Theorem 4.1. Moreover, let $F$ be defined and $w_{\varphi}$-integrable on $C[0, T]$. Then we have for $P_{X_{\tau}}$-a.e. $\vec{\xi}=\left(\xi_{0}, \xi_{1}, \cdots, \xi_{n}\right)$ $\in \mathbb{R}^{n+1}$

$$
\begin{aligned}
E_{w_{\varphi}}\left[F \mid X_{\tau}\right](\vec{\xi})= & \exp \left\{\sum _ { j = 1 } ^ { n } \frac { x _ { 0 } ( t _ { j } ) - x _ { 0 } ( t _ { j - 1 } ) } { t _ { j } - t _ { j - 1 } } \left[\left(\xi_{j}-\xi_{j-1}\right)\right.\right. \\
& \left.\left.-\frac{1}{2}\left(x_{0}\left(t_{j}\right)-x_{0}\left(t_{j-1}\right)\right)\right]\right\} E_{w_{\varphi_{\alpha}}}\left[F\left(x_{0}+\cdot\right) J \mid X_{\tau}\right]\left(\vec{\xi}-\vec{x}_{0}\right)
\end{aligned}
$$

where $J(x)=\exp \left\{-\frac{1}{2}\|h\|_{2}^{2}\right\} \exp \{-(h, x)\}$ for $x \in C[0, T]$ and $\vec{x}_{0}=\left(x_{0}\left(t_{0}\right), x_{0}\left(t_{1}\right)\right.$, $\left.\cdots, x_{0}\left(t_{n}\right)\right)$.

Proof. By Theorems 2.9 and 4.1 , we have for $P_{X_{\tau}}$-a.e. $\vec{\xi}=\left(\xi_{0}, \xi_{1}, \cdots, \xi_{n}\right) \in \mathbb{R}^{n+1}$

$$
\begin{aligned}
E_{w_{\varphi}}\left[F \mid X_{\tau}\right](\vec{\xi}) & =\int_{C} F(x-[x]+[\vec{\xi}]) d w_{\varphi}(x) \\
& =\int_{C} F\left(x_{0}+x-[x]+\left[\vec{\xi}-\vec{x}_{0}\right]\right) J(x) d w_{\varphi_{\alpha}}(x) .
\end{aligned}
$$

But we have

$$
\exp \{-(h, x)\}=\exp \left\{-\left(h, x-[x]+\left[\vec{\xi}-\vec{x}_{0}\right]\right)-\left(h,[x]-\left[\vec{\xi}-\vec{x}_{0}\right]\right)\right\}
$$


and

$$
\begin{aligned}
& \exp \left\{\left(h,\left[\vec{\xi}-\vec{x}_{0}\right]\right)\right\} \\
= & \exp \left\{\sum_{j=1}^{n} \int_{t_{j-1}}^{t_{j}} h(t) d\left[\vec{\xi}-\vec{x}_{0}\right](t)\right\} \\
= & \exp \left\{\sum_{j=1}^{n} \frac{x_{0}\left(t_{j}\right)-x_{0}\left(t_{j-1}\right)}{t_{j}-t_{j-1}}\left(\left(\xi_{j}-\xi_{j-1}\right)-\left(x_{0}\left(t_{j}\right)-x_{0}\left(t_{j-1}\right)\right)\right)\right\} .
\end{aligned}
$$

Hence we have

$$
\begin{aligned}
& E_{w_{\varphi}}\left[F \mid X_{\tau}\right](\vec{\xi}) \\
= & \int_{C} F\left(x_{0}+x-[x]+\left[\vec{\xi}-\vec{x}_{0}\right]\right) J\left(x-[x]+\left[\vec{\xi}-\vec{x}_{0}\right]\right) \exp \{-(h,[x])\} \\
& \times \exp \left\{\sum_{j=1}^{n} \frac{x_{0}\left(t_{j}\right)-x_{0}\left(t_{j-1}\right)}{t_{j}-t_{j-1}}\left(\left(\xi_{j}-\xi_{j-1}\right)-\left(x_{0}\left(t_{j}\right)-x_{0}\left(t_{j-1}\right)\right)\right)\right\} d w_{\varphi_{\alpha}}(x) \\
= & E_{w_{\varphi_{\alpha}}}\left[F\left(x_{0}+\cdot\right) J \mid X_{\tau}\right]\left(\vec{\xi}-\vec{x}_{0}\right) \exp \left\{\sum _ { j = 1 } ^ { n } \frac { x _ { 0 } ( t _ { j } ) - x _ { 0 } ( t _ { j - 1 } ) } { t _ { j } - t _ { j - 1 } } \left(\left(\xi_{j}-\xi_{j-1}\right)\right.\right. \\
& \left.\left.-\left(x_{0}\left(t_{j}\right)-x_{0}\left(t_{j-1}\right)\right)\right)\right\} \int_{C} \exp \{-(h,[x])\} d w_{\varphi_{\alpha}}(x)
\end{aligned}
$$

since $x-[x]$ and $[x]$ are independent by Theorem 2.8 , Again, by independence (Lemma 2.1), we have

$$
\begin{aligned}
& \int_{C} \exp \{-(h,[x])\} d w_{\varphi_{\alpha}}(x) \\
= & \int_{C} \exp \left\{-\sum_{j=1}^{n} \int_{t_{j-1}}^{t_{j}} h(t) d[x](t)\right\} d w_{\varphi_{\alpha}}(x) \\
= & \int_{C} \exp \left\{-\sum_{j=1}^{n} \frac{x_{0}\left(t_{j}\right)-x_{0}\left(t_{j-1}\right)}{t_{j}-t_{j-1}}\left(x\left(t_{j}\right)-x\left(t_{j-1}\right)\right)\right\} d w_{\varphi_{\alpha}}(x) \\
= & \prod_{j=1}^{n}\left[\left[\frac{1}{2 \pi\left(t_{j}-t_{j-1}\right)}\right]^{\frac{1}{2}} \int_{\mathbb{R}} \exp \left\{-\frac{x_{0}\left(t_{j}\right)-x_{0}\left(t_{j-1}\right)}{t_{j}-t_{j-1}} v_{j}-\frac{1}{2\left(t_{j}-t_{j-1}\right)} v_{j}^{2}\right\} d v_{j}\right] \\
= & \exp \left\{\sum_{j=1}^{n} \frac{\left(x_{0}\left(t_{j}\right)-x_{0}\left(t_{j-1}\right)\right)^{2}}{2\left(t_{j}-t_{j-1}\right)}\right\}
\end{aligned}
$$

so that we have

$$
\begin{aligned}
E_{w_{\varphi}}\left[F \mid X_{\tau}\right](\vec{\xi})= & \exp \left\{\sum _ { j = 1 } ^ { n } \frac { x _ { 0 } ( t _ { j } ) - x _ { 0 } ( t _ { j - 1 } ) } { t _ { j } - t _ { j - 1 } } \left[\left(\xi_{j}-\xi_{j-1}\right)\right.\right. \\
& \left.\left.-\frac{1}{2}\left(x_{0}\left(t_{j}\right)-x_{0}\left(t_{j-1}\right)\right)\right]\right\} E_{w_{\varphi_{\alpha}}}\left[F\left(x_{0}+\cdot\right) J \mid X_{\tau}\right]\left(\vec{\xi}-\vec{x}_{0}\right)
\end{aligned}
$$

which is the desired result. 
Letting $\alpha=0$ in Theorem 4.2, we have the following corollary from the theorem since $\varphi_{\alpha}=\varphi$.

Corollary 4.3. Under the assumptions and notations given as in Theorem 4.2 with one exception $\alpha=0$, we have $x_{0}(t)=\int_{0}^{t} h(s) d s$ for $t \in[0, T]$ and

$$
\begin{aligned}
E_{w_{\varphi}}\left[F \mid X_{\tau}\right](\vec{\xi})= & \exp \left\{\sum _ { j = 1 } ^ { n } \frac { x _ { 0 } ( t _ { j } ) - x _ { 0 } ( t _ { j - 1 } ) } { t _ { j } - t _ { j - 1 } } \left[\left(\xi_{j}-\xi_{j-1}\right)\right.\right. \\
& \left.\left.-\frac{1}{2}\left(x_{0}\left(t_{j}\right)-x_{0}\left(t_{j-1}\right)\right)\right]\right\} E_{w_{\varphi}}\left[F\left(x_{0}+\cdot\right) J \mid X_{\tau}\right]\left(\vec{\xi}-\vec{x}_{0}\right) .
\end{aligned}
$$

For notational convenience, let $E_{m_{w}}$ denote the (conditional) Wiener integral on $C_{0}[0, T]$.

Under the assumption that $V$ is a non-negative continuous function on $\mathbb{R}$ satisfying the condition

$$
\int_{\mathbb{R}} V\left(\xi_{1}\right) \exp \left\{-\frac{\xi_{1}^{2}}{2 T}\right\} d \xi_{1}<\infty
$$

for every $T>0$, we have for a.e. $\xi_{1} \in \mathbb{R}$

$$
\begin{aligned}
& E_{w_{\varphi}}\left[\exp \left\{-\int_{0}^{T} V(x(s)) d s\right\} \mid x\left(t_{0}\right)=0, x(T)=\xi_{1}\right] \\
= & E_{m_{w}}\left[\exp \left\{-\int_{0}^{T} V(x(s)) d s\right\} \mid x(T)=\xi_{1}\right]
\end{aligned}
$$

and for a.e. $\left(\xi_{1}, \cdots, \xi_{n}\right) \in \mathbb{R}^{n}$

$$
\begin{aligned}
& E_{w_{\varphi}}\left[\exp \left\{-\int_{0}^{T} V(x(s)) d s\right\} \mid x\left(t_{0}\right)=0, x\left(t_{1}\right)=\xi_{1}, \cdots, x\left(t_{n}\right)=\xi_{n}\right] \\
= & E_{m_{w}}\left[\exp \left\{-\int_{0}^{T} V(x(s)) d s\right\} \mid x\left(t_{1}\right)=\xi_{1}, \cdots, x\left(t_{n}\right)=\xi_{n}\right]
\end{aligned}
$$

by Theorems 2.4 and 2.9 .

In [10, Yeh showed that the function $U$ defined on $\mathbb{R} \times(0, \infty)$ by

$$
\begin{aligned}
& U\left(\xi_{1}, T\right) \\
= & \left(\frac{1}{2 \pi T}\right)^{\frac{1}{2}} \exp \left\{-\frac{\xi_{1}^{2}}{2 T}\right\} E_{m_{w}}\left[\exp \left\{-\int_{0}^{T} V(x(s)) d s\right\} \mid x(T)=\xi_{1}\right]
\end{aligned}
$$

satisfies the Kac-Feynman integral equation

$$
\begin{array}{r}
U\left(\xi_{1}, T\right)=\left(\frac{1}{2 \pi T}\right)^{\frac{1}{2}} \exp \left\{-\frac{\xi_{1}^{2}}{2 T}\right\}-\int_{0}^{T}\left[\frac{1}{2 \pi(T-s)}\right]^{\frac{1}{2}} \int_{\mathbb{R}} \exp \left\{-\frac{\left(\xi_{1}-\eta\right)^{2}}{2(T-s)}\right\} \\
\times V(\eta) U(\eta, s) d \eta d s
\end{array}
$$

whose solution is given by

$$
U\left(\xi_{1}, T\right)=\sum_{k=0}^{\infty}(-1)^{k} U_{k}\left(\xi_{1}, T\right), \quad\left(\xi_{1}, T\right) \in \mathbb{R} \times(0, \infty),
$$


where the sequence $\left\{U_{k}\right\}_{k=0}^{\infty}$ is defined inductively by

$$
\begin{gathered}
U_{0}\left(\xi_{1}, T\right)=\left(\frac{1}{2 \pi T}\right)^{\frac{1}{2}} \exp \left\{-\frac{\xi_{1}^{2}}{2 T}\right\}, \\
U_{k+1}\left(\xi_{1}, T\right)=\int_{0}^{T}\left[\frac{1}{2 \pi(T-s)}\right]^{\frac{1}{2}} \int_{\mathbb{R}} \exp \left\{-\frac{\left(\xi_{1}-\eta\right)^{2}}{2(T-s)}\right\} V(\eta) U_{k}(\eta, s) d \eta d s
\end{gathered}
$$

for $k=0,1, \cdots$. Thus $E_{w_{\varphi}}\left[\exp \left\{-\int_{0}^{T} V(x(s)) d s\right\} \mid x\left(t_{0}\right)=0, x(T)=\xi_{1}\right]$ can be found using (1) and (3).

Now, for $\xi_{1}, \xi_{2} \in \mathbb{R}$ and $t \in(0, T]$, let

$$
\begin{aligned}
& U\left(\xi_{1}, \xi_{2}-\xi_{1}, T-t\right) \\
= & {\left[\frac{1}{2 \pi(T-t)}\right]^{\frac{1}{2}} \exp \left\{-\frac{\left(\xi_{2}-\xi_{1}\right)^{2}}{2(T-t)}\right\} } \\
& \times E_{w_{\varphi}}\left[\exp \left\{-\int_{t}^{T} V(x(s)) d s\right\} \mid x\left(t_{0}\right)=0, x(t)=\xi_{1}, x(T)=\xi_{2}\right] .
\end{aligned}
$$

Using the same process in [6. pp. 388-389], (4) satisfies the integral equation

$$
\begin{aligned}
& U\left(\xi_{1}, \xi_{2}-\xi_{1}, T-t\right) \\
= & {\left[\frac{1}{2 \pi(T-t)}\right]^{\frac{1}{2}} \exp \left\{-\frac{\left(\xi_{2}-\xi_{1}\right)^{2}}{2(T-t)}\right\}-\int_{0}^{T-t}\left[\frac{1}{2 \pi(T-t-u)}\right]^{\frac{1}{2}} } \\
& \times \int_{\mathbb{R}} \exp \left\{-\frac{\left(\xi_{2}-\gamma\right)^{2}}{2(T-t-u)}\right\} V(\gamma) U\left(\xi_{1}, \gamma-\xi_{1}, u\right) d \gamma d u
\end{aligned}
$$

by (2) so that we have

$$
U\left(\xi_{1}, \xi_{2}-\xi_{1}, T-t\right)=\sum_{k=0}^{\infty}(-1)^{k} U_{k}\left(\xi_{2}, \xi_{2}-\xi_{1}, T-t\right)
$$

where

$$
U_{0}\left(\xi_{1}, \xi_{2}-\xi_{1}, T-t\right)=\left[\frac{1}{2 \pi(T-t-u)}\right]^{\frac{1}{2}} \exp \left\{-\frac{\left(\xi_{2}-\xi_{1}\right)^{2}}{2(T-t)}\right\}
$$

and

$$
\begin{aligned}
& U_{k+1}\left(\xi_{1}, \xi_{2}-\xi_{1}, T-t\right) \\
= & \int_{0}^{T-t}\left[\frac{1}{2 \pi(T-t-u)}\right]^{\frac{1}{2}} \int_{\mathbb{R}} \exp \left\{-\frac{\left(\xi_{2}-\gamma\right)^{2}}{2(T-t-u)}\right\} V(\gamma) U_{k}\left(\xi_{1}, \gamma-\xi_{1}, u\right) d \gamma d u
\end{aligned}
$$

for $k=0,1,2, \cdots$. In particular, the solution $U\left(\xi_{1}, T\right)$ of the Kac-Feynman integral equation is a special case, namely,

$$
U\left(\xi_{1}, T\right)=U\left(0, \xi_{1}, T\right)
$$


We are now ready to write out an expression for the multi-conditional expectation. Using (4.6) in [6], (11) and (2), we can write for a.e. $\left(\xi_{1}, \cdots, \xi_{n}\right) \in \mathbb{R}^{n}$

$$
\begin{aligned}
& E_{w_{\varphi}}\left[\exp \left\{-\int_{0}^{T} V(x(s)) d s\right\} \mid x\left(t_{0}\right)=0, x\left(t_{1}\right)=\xi_{1}, \cdots, x\left(t_{n}\right)=\xi_{n}\right] \\
= & \prod_{j=1}^{n}\left[2 \pi\left(t_{j}-t_{j-1}\right)\right]^{\frac{1}{2}} \exp \left\{\frac{\left(\xi_{j}-\xi_{j-1}\right)^{2}}{2\left(t_{j}-t_{j-1}\right)}\right\} U\left(\xi_{j-1}, \xi_{j}-\xi_{j-1}, t_{j}-t_{j-1}\right)
\end{aligned}
$$

where $\xi_{0}=0$ and $t_{n}=T$.

\section{REFERENCES}

[1] R. B. Ash, Real analysis and probability, Academic Press, New York-London, 1972. MR0435320 (55:8280)

[2] R. H. Cameron and W. T. Martin, Transformations of Wiener integrals under translations, Ann. Math. 45 (1944), 386-396. MR0010346 (6:5f)

[3] K. S. Chang and J. S. Chang, Evaluation of some conditional Wiener integrals, Bull. Korean Math. Soc. 21 (1984), no. 2, 99-106. MR768465 (86e:28018)

[4] M. K. Im and K. S. Ryu, An analogue of Wiener measure and its applications, J. Korean Math. Soc. 39 (2002), no. 5, 801-819. MR1920906 (2003g:28028)

[5] R. G. Laha and V. K. Rohatgi, Probability theory, John Wiley \& Sons, New York-ChichesterBrisbane, 1979. MR.534143 (80k:60001)

[6] C. Park and D. L. Skoug, A simple formula for condtitional Wiener integrals with applications, Pacific J. Math. 135 (1988), no. 2, 381-394. MR968620 (90c:28022)

[7] K. S. Ryu and M. K. Im, A measure-valued analogue of Wiener measure and the measurevalued Feynman-Kac formula, Trans. Amer. Math. Soc. 354 (2002), no. 12, 4921-4951. MR.1926843 (2004b:28020)

[8] J. Yeh, Stochastic processes and the Wiener integral, Marcel Dekker, New York, 1973. MR 0474528 (57:14166)

[9] J. Yeh, Inversion of conditional expectations, Pacific J. Math. 52 (1974), 631-640. MR0365644 (51:1896)

[10] J. Yeh, Inversion of conditional Wiener integrals, Pacific J. Math. 59 (1975), no. 2, 623-638. MR0390162 (52:10988)

[11] J. Yeh, Transformation of conditional Wiener integrals under translation and the CameronMartin translation theorem, Tohôku Math. J. 30 (1978), no. 4, 505-515. MR516883 (80e:60094)

Department of Mathematics, Kyonggi University, Kyonggido Suwon 443-760, Korea

E-mail address: j94385@kyonggi.ac.kr 